\title{
Bello y las "escrituras disciplinarias": diccionarios, gramáticas, ortografías
}

\author{
Barry L. Velleman* \\ Marquette University, EE. UU.
}

\begin{abstract}
Resumen
Los diccionarios y las gramáticas representan herramientas de planificación lingüística, y, junto con las constituciones y los manuales de urbanidad, son "escrituras disciplinarias" (González Stephan 1995). La "etnortografía", subdisciplina de la glotopolítica, es otra variedad de "escritura disciplinaria", puesto que las iniciativas de reforma ortográfica pueden reflejar y promover determinadas ideologías políticas, culturales y religiosas (Calero Vaquera 2010). En sus propuestas destinadas a simplificar la ortografía castellana, Andrés Bello (1781-1865) y Domingo Sarmiento (1811-1888) son representantes de diversas orientaciones etnortográficas. Se interpreta a Bello como figura esencial del cultivo de la cultura latinoamericana poscolonial desde la perspectiva del modelo de los llamados "nacionalismos entrelazados".
\end{abstract}

Palabras clave: Andrés Bello, Domingo F. Sarmiento, ortografía, historia cultural latinoamericana, nacionalismos entrelazados.

* Para correspondencia, dirigirse a: Barry L. Velleman (barry.velleman@marquette.edu), Foreign Languages and Literatures, Marquette University, Milwaukee, WI 53233, USA / EE. UU. 


\title{
Bello and 'Disciplinary Texts': Dictionaries, Grammars, ORTHOGRAPHIES
}

\begin{abstract}
Dictionaries and grammars represent tools of language planning and, along with constitutions and etiquette guides, are "disciplinary texts" (González Stephan 1995). "Ethno-orthography", a sub-discipline of glottopolitics, is another kind of "disciplinary text", since orthographic reform initiatives may reflect and promote particular political, cultural, and religious ideologies (Calero Vaquera 2010). In their proposals designed to simplify Spanish spelling, Andrés Bello (1781-1865) and Domingo Sarmiento (1811-1888) are representatives of differing ethno-orthographic orientations. Bello is seen as an essential figure in the cultivation of Spanish American post-colonial culture from the perspective of the model known as "interlocking nationalisms".
\end{abstract}

Key words: Andrés Bello, Domingo F. Sarmiento, orthography, Spanish American cultural history, interlocking nationalisms.

Recibido: 13/03/14 Aceptado: $10 / 04 / 14$

\section{EL DICCIONARIO Y LAS 'ESCRITURAS DISCIPLINARIAS'}

En su obra teatral Translations (Traducciones, 1980), el dramaturgo irlandés Brian Friel (n. 1929) traza la historia de Baile Beag, una comunidad de habla irlandesa en la primera parte del siglo XIX. Allí, a raíz de una encuesta cartográfica ordenada por el gobierno inglés, todos los topónimos locales tenían que ser registrados para traducirlos al inglés. Lo que parece ser un acto puramente administrativo pasa a iluminar lo profundo de la relación entre los ruidos del lenguaje y la realidad histórica que estos representan. Uno de los personajes, Hugh, encuentra en el piso el libro oficial de nombres, y se pone a leer los extraños nombres nuevos. Su hijo Owen le quita el libro y le dice: "Yo tomo eso. Es solamente un catálogo de nombres" (Friel 1981: 87; todas las traducciones al español son mías a menos que se indique a otro/a traductor[a]: BLV).

El aporte lingüístico y cultural del interés de Andrés Bello en los diccionarios se manifiesta en numerosos aspectos: en sus glosas a los nombres 
de plantas o frutas americanas en la Silva a la agricultura de la zona tórrida o a los arcaísmos de la Crónica del Cid; en sus meditadas reflexiones sobre diccionarios tan variados como el de la Academia, el de galicismos de Baralt, y otros más especializados; en la petición de Salvá de que el venezolano le sirviera de informante sobre los americanismos (Pérez 2013). El estudio teórico de la lexicografía, como reflejo de la cultura, demuestra que un diccionario no es solamente "un catálogo de nombres". Es un documento que refleja la realidad social tanto de la comunidad lingüística como de los compiladores.

Cargados con un ingrediente ideológico y afectivo y respondiendo a los vicios de prácticas discursivas manipulatorias, los diccionarios están ofreciendo una visión del mundo y un conocimiento cercano y penetrante de las actitudes mentales de las sociedades e individuos que se muestran reflejados en las cosas referencializadas y en las cosas aludidas o dichas en la descripción diccionariológica (Pérez 2000: 23).

Con el Diccionario (1755) de Samuel Johnson (1709-1784) -obra que poseía Bello, en su biblioteca particular, en la edición de 1818 (Velleman 1995: 198) - el papel del diccionario cambió. Antes de Johnson, el diccionario tenía como objetivo la enumeración de "palabras difíciles, anticuadas, extranjeras, exóticas y técnicas" para que las clases menos letradas pudieran estar informadas sobre el lenguaje de la sociedad (Annoni 2010). Al incluir las palabras comunes al lado de las difíciles, Johnson produjo una imagen comprensiva del idioma y de la cultura británica implícita en su selección, definición y ejemplificación.

Después de Johnson, los diccionarios adquirieron un rol cultural nuevo $\mathrm{y}$, con los atlas y la enciclopedia, los museos y otras instituciones, se convirtieron en modos de recoger y definir familias de objetos culturales. Por consiguiente, se hicieron asimismo maneras de determinar identidades culturales lingüísticas y nacionales (Annoni 2010) ${ }^{1}$.

El diccionario puede ser también, como la gramática de una lengua, una herramienta de planificación lingüística. Según Kaplan y Baldauf (1997: 66), las gramáticas y los diccionarios son las herramientas principales

\footnotetext{
Revela mucho la definición que da Johnson para la palabra oats 'avena': "un grano que en Inglaterra se les da generalmente a los caballos, pero que en Escocia mantiene a la gente" (citado en Winchester 1998: 95).
} 
para la estandarización lingüística ${ }^{2}$. González Stephan (1995) ha escrito sobre las "escrituras disciplinarias", textos de mission civilisatrice como las gramáticas, las constituciones y los manuales de urbanidad ${ }^{3}$. Pérez ha señalado que el "Prólogo" a la Gramática de Bello constituye "el primer texto doctrinal metalingüístico hispanoamericano", que define los fundamentos de una "gramática nacional y particular", una gramática americana que acepta pero que expande la tradición, "purismo y progreso" (1998: 73-74). Nos encontramos con la figura de Bello, entonces, en esta dirección de planificación lingüística.

\section{LA PLANIFICACIÓN LINGÜÍSTICA Y LA PRESERVACIÓN DE LA LENGUA}

Kaplan y Baldauf (1997) definen la planificación lingüística como "ideas, leyes y reglamentos (política lingüística), normas de cambio, creencias y prácticas destinadas a conseguir un cambio planificado (o a impedir que se produzca un cambio) en el uso lingüístico de una o más comunidades" (citado y traducido en del Valle y Gabriel-Stheeman 2004: 26). Si utilizamos los conceptos de Haugen (1972) sobre la estandarización (véase del Valle y Gabriel-Stheeman 2004: 26-27), Bello contribuyó a los procesos de la selección de una norma lingüística y de la codificación de la misma mediante la fijación de esa norma. Bello también fomentó la elaboración y la aceptación del estándar escogido - su expansión y su uso voluntario y natural en muchos contextos sociales. Kaplan y Baldauf distinguen entre dos tipos de "preservación" de una lengua: la de la comunidad, en la que se ve amenazada una lengua por la extinción, y la dominante, en que el uso lingüístico tiene que mantenerse para evitar que se desvíe de una norma estándar aceptada (1997: 78). Esta segunda variedad de "preservación" caracteriza a la obra lingüística de Bello en Chile, obra motivada por las

2 Para Bello, estos dos tipos de texto se complementan: el diccionario "da el significado de las raíces", mientras que la gramática expone "el valor de las inflexiones [morfología] y combinaciones [sintaxis]" (Bello 1981b [1847]: 127).

3 Es evidente la relación entre el uso lingüístico y la urbanidad si consideramos conceptos como este: "El habla es el regalo que distingue al hombre de los animales y que hace posible la sociedad" (Aster 1863: 70). Vid. unas obras del siglo XVIII que incorporaban el entrenamiento lingüístico, ortográfico y caligráfico con la cortesía, como la del Padre Santiago Delgado (1799). Sobre Bello y la "gramática de las costumbres", vid. Straka (2006). 
necesidades inmediatas educativas, legislativas, sociales y culturales de una nación en vías de desarrollo y también, según el famoso "Prólogo" de su Gramática (1847), por el temor de la posible fragmentación lingüística del español en las nuevas repúblicas americanas.

Un modelo útil para describir y evaluar la contribución de Bello se ve en la matriz del cultivo de la cultura según la teoría de interlocking nationalisms ("nacionalismos entrelazados") elaborada por The Study Platform on Interlocking Nationalisms o SPIN ("La plataforma de estudio de los nacionalismos entrelazados": www.spinnet.eu). SPIN tiene como objetivo el estudio de las raíces del sistema cultural e histórico de los nacionalismos europeos. La matriz consiste en una serie de actividades e iniciativas que representan marcadores del nacionalismo cultural, distinguiendo entre cuatro campos culturales (lengua, discurso y literatura, artefactos culturales, prácticas culturales). Limitándonos a las categorías más aplicables a la contribución de Bello y a la cultura pos-revolucionaria, es posible identificar estas áreas de actividad cultural y sus productos y prácticas según las tres etapas de desarrollo propuestas por la matriz. En la primera etapa, "recuperación/inventario", se pone énfasis en el (re)descubrimiento y clasificación del material cultural, sea lingüístico, literario, legal, histórico, etc. La segunda etapa es "el cultivo y la perpetuación" del material, actualizándolo, procesos en los que se investigan diversas aproximaciones y orientaciones, contribuyendo a posibles "polémicas". En la tercera etapa, se propaga el material cultural en la esfera pública mediante la planificación, la educación y la conmemoración de contribuciones culturales.

En el campo cultural denominado "lengua" se proponen (1) la creación de documentos tales como los diccionarios, las gramáticas y las ortografías, y (2) los debates sobre el "purismo" lingüístico y la estandarización. En el dominio público de la "lengua", encontramos la planificación y la educación lingüística. Bajo "discurso/literatura" la matriz coloca, para la etapa "recuperación", las ediciones de textos antiguos (literarios, históricos y legales); para la etapa "cultivo/propagación", las traducciones y adaptaciones de la literatura y la historiografía nacionales; y para la etapa "proclamación en la esfera pública", encontramos la educación sobre la historia y las conmemoraciones y las competencias literarias.

Además de las iniciativas sobre la lengua y la literatura, la matriz propone dos áreas adicionales: el ambiente social (agrupaciones académicas como las asociaciones de estudiosos, los congresos y las sociedades de lectura; y productos culturales como las publicaciones periódicas) y la infraestructura institucional (entidades más o menos estables como las universidades, las cátedras, las bibliotecas, los archivos, los museos, las academias y las agencias gubernamentales). Se presenta a continuación la matriz de SPIN, 
adaptada para reflejar unas posibilidades del cultivo de la cultura poscolonial.

Cuadro 1. El cultivo de la cultura: una matriz ${ }^{4}$

\begin{tabular}{|c|c|c|c|}
\hline preocupaciones & $\begin{array}{c}\text { recuperación/ } \\
\text { inventario }\end{array}$ & $\begin{array}{c}\text { cultivo/ } \\
\text { perpetuación/ } \\
\text { inspiración }\end{array}$ & $\begin{array}{l}\text { propagación/ } \\
\text { proclamación en } \\
\text { la esfera pública }\end{array}$ \\
\hline $\begin{array}{l}\text { campos } \\
\text { culturales }\end{array}$ & 1 & 2 & 3 \\
\hline lengua & $\begin{array}{l}\text { diccionarios, } \\
\text { gramáticas }\end{array}$ & $\begin{array}{l}\text { ortografía, } \\
\text { debates sobre la } \\
\text { estandarización } \\
\text { y los dialectos, } \\
\text { purismo } \\
\text { lingüístico }\end{array}$ & $\begin{array}{l}\text { activismo lingüístico, } \\
\text { planificación } \\
\text { lingüística, } \\
\text { educación sobre } \\
\text { la lengua }\end{array}$ \\
\hline $\begin{array}{l}\text { discursol } \\
\text { literatura }\end{array}$ & $\begin{array}{l}\text { ediciones de } \\
\text { textos antiguos } \\
\text { (literarios, } \\
\text { históricos, } \\
\text { legales) }\end{array}$ & \begin{tabular}{|l|} 
traducciones y \\
adaptaciones, \\
literatura nacional, \\
historiografía \\
nacional, crítica \\
literaria/cultural \\
\end{tabular} & $\begin{array}{l}\text { educación sobre } \\
\text { la historia, } \\
\text { conmemoraciones, } \\
\text { eventos/premios/ } \\
\text { festivales literarios }\end{array}$ \\
\hline ambiente social & \multicolumn{3}{|c|}{$\begin{array}{l}\text { asociaciones, congresos, sociedades de lectura; publicaciones } \\
\text { periódicas }\end{array}$} \\
\hline $\begin{array}{l}\text { infraestructura } \\
\text { institucional }\end{array}$ & \multicolumn{3}{|c|}{$\begin{array}{l}\text { universidades, cátedras, bibliotecas, archivos, museos, academias, } \\
\text { agencias gubernamentales }\end{array}$} \\
\hline
\end{tabular}

De estas áreas, hay muy pocas no tocadas por la inmensa obra de Bello: su Gramática, su participación en discusiones sobre la ortografía, la estandarización y el papel de la Academia; sus estudios medievales y sus ediciones de la literatura antigua española y del derecho romano; su producción en las publicaciones periódicas de Caracas, Londres y Santiago; su crítica literaria e historiográfica; su contribución a la educación popular, a las escuelas normales y al gobierno; su fundación y organización de la Universidad de Chile; y, por ese medio y otros, su fomento de investigaciones históricas, literarias y científicas.

4 Adaptación del esquema de SPIN (http://www.spinnet.eu/mapping). 


\section{LA ETNORTOGRAFÍA}

Calero Vaquera (2010) ha propuesto el término "etnortografía" para referirse a la subdisciplina de la glotopolítica que representa la aplicación de las "ideologías" a los manuales de ortografía (2010). Calero Vaquera acepta la definición de la ideología propuesta por van Dijk (1998: 21):

... la base de las representaciones sociales compartidas por los miembros de un grupo. Esto significa que las ideologías les permiten a las personas, como miembros de un grupo, organizar la multitud de creencias sociales acerca de lo que sucede, bueno o malo, correcto o incorrecto, según ellos, y actuar en consecuencia (Calero Vaquera 2010: 33).

El propósito de Calero Vaquera es el de mostrar que la "ideología" se encontraba no solamente en los diccionarios y en las gramáticas, sino también en los manuales de ortografía. La etnortografía, por consiguiente, es el correlato gráfico de la etnolexicografía y la etnogramática. Narvaja de Arnoux ha documentado la "ideología" de las gramáticas de Bello escritas para las escuelas a mediados del siglo XIX, cuyos ejemplos evidencian una "penetración del discurso religioso" además del discurso moral y patriótico (Narvaja de Arnoux 2008: 290). Así es como en el "Compendio de gramática castellana escrito para el uso de las escuelas primarias" (Bello 1981c [1862]) encontramos ejemplos como Dios, ángel (los primeros ejemplos de sustantivos, 237), Dios se compadece de los pecadores (241), En medio de tantos peligros, imploremos sobre nuestra patria la protección del Dios de las misericordias (249), etc.

Para el tiempo estudiado por Calero Vaquera en su artículo de 2010, mediados del siglo XX, ya había pasado la época de los reformadores de la ortografía, y otra vez hay que buscar la ideología sociocultural en los ejemplos ofrecidos ${ }^{5}$. Pero recordemos que en 1823 Bello publicó, en coautoría con Juan García del Río (1794-1854), un artículo en la Biblioteca americana sobre un "nuevo sistema de ortografía para América" (García del Río y Bello 1972 [1823]). Las circunstancias de la composición de este artículo sugieren otra interpretación de la "etnortografía" en el contexto poscolonial. Esta interpretación tiene dos fuentes y resultados deseados. Primero, la modernización de la ortografía es una expresión de una

\footnotetext{
5 El manual ortográfico analizado por la investigadora española es la del uruguayo Nicasio H. García (1947).
} 
necesidad poscolonial de educación. El segundo, más radical y consistente con un concepto cultural nacionalista romántico, representa un esfuerzo por establecer una identidad nacional poscolonial rompiendo con los modelos imperiales anteriores. Es útil considerar estas dos interpretaciones al estudiar la participación de Andrés Bello y Domingo Faustino Sarmiento (1811-1888) en lo que se ha llamado la "polémica sobre la ortografía".

\section{BELLO Y SARMIENTO}

A diferencia del Sarmiento de los años 1842-1849, Bello le tiene miedo a cualquier sugerencia de escisión del español de América respecto del de España (Rosenblat 1981: cvii). No hay en Bello "la prédica de una independencia idiomática que viniera a completar a la política, como desde sus tiempos han venido reclamando algunos escritores de nacionalismo especialmente susceptible en la Argentina, en Brasil y en Norteamérica" (Alonso 1972 [1951]: xvi). Ángel Rosenblat (1981: lxxxix, n. 102) ha sugerido que una oración del escrito de 1823 -la declaración más fuertemente antiespañola del artículo- fue de García del Río:

... [E]s necesario confesar que en la jeneralidad de los habitantes de América no se encontraban cinco personas en ciento que poseyesen gramaticalmente su propia lengua, i apénas una que la escribiese correctamente. Tal era el efecto del plan adoptado por la corte de Madrid respecto de sus posesiones coloniales, i aun la consecuencia necesaria del atraso en que se encontraba la misma España (1972 [1823]: 50; reproducimos la ortografía original).

Mientras tanto, es probablemente Bello el que atribuye la deficiencia principal de la Real Academia de Madrid a su necesaria organización como grupo: "Pero debemos tener presente que las operaciones de un cuerpo de esta especie no pueden ser tan sistemáticas, ni tan fijos sus principios, como los de un individuo" (García del Río y Bello 1972 [1823]: 52) .

6 Cf. Weinstein (1982: 105-106): "No se puede estudiar la planificación lingüística sin referencia a iniciativas individuales". Al considerar el diccionario académico, Bello sugiere otro factor que explica unas de sus fallas, la enormidad de la tarea: en este diccionario "se conservan $[\ldots]$ algunos errores; sin duda porque en una obra tan vasta es imposible revisar artículo por artículo" (Bello 1981a: 121). 
Si Bello enfatiza principalmente la practicidad educativa de las modificaciones ortográficas, como Sarmiento -quien era director de la enseñanza primaria de Chile- este va más lejos en proponer una ortografía como la "aserción simbólica de la identidad pos-colonial". Esta iconización les da a las opciones ortográficas un significado social y político (vid. Sebba 2007).

Las ortografías del español típicamente han obedecido a alguna meta social, sea educativa, patriótica o separatista. Para Nebrija, la uniformidad del sistema ortográfico (que idealmente corresponderá exactamente a la pronunciación) es paralela a la codificación nacionalista del idioma en su gramática. En 1609 Mateo Alemán (1547-¿1615?) publicó en México su Ortografía castellana para que "el bien escrevir" se aprendiera en todo el mundo hispánico: el "mal escrevir" "nos infama cerca de otras naciones" (citado en Rosenblat 1981: xliii-xliv): uniformidad imperial y orgullo nacional. Uno de los sistemas más radicales es el de la Ortografía kastellana, nueva i perfeta (1630) de Gonzalo Correas (1571-1631), obra cuya dedicatoria le implora al Rey que venza a los latinizantes: "V. M. [...] haga kon su mano poderosa esta merzed a la tierna edad i a toda España i a su lengua natural para que salga de la esklavitud en ke la tienen los ke estudian latin" (citado en Rosenblat 1981: xlviii) ${ }^{7}$.

Para los lingüistas antropológicos del siglo XX, quienes trabajaban muchas veces con lenguas que carecían de forma escrita, una ortografía parecía ser solamente un sistema mecánico según un "principio fonémico" que dejaba al lado cualquier consideración sociolingüística. Bajo esta interpretación, un sistema ortográfico era "un catálogo de grafías", carente de significado social, tal como un diccionario parece ser a primera vista "un catálogo de nombres". No obstante, como descubrió el lingüista checo-norteamericano Paul Garvin (1919-1994) cuando intentaba crear una ortografía para el ponapeano (un idioma del Pacífico), "[e]l problema de diseñar un sistema ortográfico aceptable, lo que inicialmente puede haber parecido un asunto puramente, o por lo menos principalmente, lingüístico, al verlo de más cerca se convirtió en un problema de lengua y cultura por excelencia" (Garvin 1954, citado en Sebba 2012: 2).

\footnotetext{
La grafía $k$ fue símbolo de la antilatinización del sistema ortográfico para muchos de estos escritores. Más recientemente ha sido símbolo de protesta contra las reglas ortográficas del español, síntoma del rechazo de muchas normas sociales entre los anarquistas o separatistas de Cataluña y del País Vasco, entre los activistas mapuche (Kropff 2005: 122-123), etc. La grafía $k$ servía el mismo propósito en las Islas Filipinas, donde su uso en el tagalo representaba una protesta contra el dominio español. Vid. Sebba 2007.
} 
Para Sarmiento especialmente, la ortografía venía a ser un gravísimo "problema de lengua y cultura" y uno de los numerosos campos de la batalla de emancipación. Su Memoria sobre ortografia americana (1843) ataca a la cultura española de la que es parte la Academia, "destronada, Real y estranjera" (2007 [1843]: 26); la aceptación de las reglas de dicha corporación es "abyección indigna de naciones que han asumido el rango de tales" (2007 [1843]: 25). Sarmiento estaría contento con dos sistemas ortográficos: al lado de una ortografía docta, específicamente para los sabios en que "mostremos que recordamos haber sido españoles, que sabemos de donde procede el castellano y como lo hablan en la península", Sarmiento propone otra "vulgar, ignorante, americana para que en ella aprendan á leer en cuatro días nuestros hijos" (2007 [1843]: 27).

En un importante estudio, el crítico francés Paul Verdevoye (1912-2001) describe la "polémica acerca del idioma" entre Bello y Sarmiento, diciendo: "Al hacer de un problema lingüístico una cuestión política, Sarmiento lleva la discusión a un terreno adonde el venezolano no quiere seguirlo" (1981: 105). Esta diferencia de orientación, expresada de acuerdo con la obvia diferencia de temperamento, se ha visto como reflejo del conflicto generacional entre dos posturas fundamentalmente distintas. Blanco (1994) ha diferenciado entre la actitud "castizo/purista", que apoyaba la "purificación" del español en América, y la actitud "rupturista", que veía la lengua como símbolo y realización de la identidad nacional, rechazando por lo tanto el idioma de la madre patria. La tercera actitud postulada por Blanco es la del "equilibrio" entre el "purismo" y la "ruptura", es decir, una perspectiva que acepta el idioma heredado como base y también la posibilidad (y la necesidad) de modificaciones locales, actitud expresada por Bello en el "Prólogo" de su Gramática, sin sugerir la existencia de normas policéntricas.

Según Narvaja de Arnoux, las propuestas ortográficas de Bello y de Sarmiento se inscriben "en un emprendimiento más amplio [que la mera ortografía] que es el desarrollo de la cultura hispanoamericana moderna, para lo cual la marcación en la lengua escrita es la señal de cambio" (2008: 185). Verdevoye ha argüido que, a pesar de las polémicas, en realidad este tema, la cultura hispanoamericana moderna, motivaba a los dos y que, específicamente en relación con sus ideas sobre la lengua, "no es imposible que el pensamiento lingüístico de Sarmiento hubiese evolucionado al contacto de Andrés Bello" (1981: 107).

Unos escritores han notado el paralelismo entre unos elementos de la controversia chilena sobre la ortografía, como parte de la construcción de naciones, y la obra del norteamericano Noah Webster (1758-1843: vid., por ejemplo, Payàs 2008: 18-19). No existe evidencia de que Bello conociera la obra neográfica de Webster, pero hay interesantes paralelismos entre los 
dos. Ambos compartían una temprana afición al derecho; las motivaciones ortográficas de los dos eran similares; ninguno de los dos ponía en práctica todas las innovaciones ortográficas que había recomendado; y ambos escritores terminaron aceptando, con algunas excepciones, la ortografía convencional (Cooper 1989: 147; Weinstein 1982: 97). Por casualidad, Bello y Webster estaban en Londres en 1825, aunque no hay evidencia de contacto personal entre los dos (Payàs 2008: 19). Lo seguro es que el discurso independentista de Webster se parece más al de Sarmiento que al de Bello. Jaksic ha señalado que le interesaba mucho más a Webster que a Bello "reivindicar la peculiaridad de la nueva nación [...] para así legitimar su separación de Europa" (2007: 274). Como Sarmiento, Webster critica, en términos sumamente severos, a los escritores y la política de la "madre patria". Para el lexicógrafo norteamericano, Europa no podía servir de modelo cultural por haberse "envejecido en tontería, corrupción y tiranía" (citado en Payàs 2008: 18). La Gran Bretaña, "cuyos hijos somos, no debería ya ser nuestro modelo; el gusto de sus escritores es ya corrupto, y su lengua está en decadencia”. Webster utiliza casi las mismas palabras que utilizará 54 años después Sarmiento cuando recomienda que "aprovechemos [nosotros los norteamericanos] el momento presente y establezcamos una lengua nacional, así como un gobierno nacional" (citado y traducido en Payàs 2008: $18-19$, n. 9 y 10$)^{8}$.

\section{CONCLUSIÓN}

En este breve estudio hemos intentado esbozar una parte de la inmensa contribución cultural de Andrés Bello mediante unos modelos específicos, clarificando en el proceso la posibilidad de añadir las ortografías a los textos "disciplinarios" como vehículo de los discursos etnográficos. La ortografía, como el diccionario, la gramática, el derecho, la educación popular y la universidad, temas todos íntimamente relacionados con las preocupaciones americanistas del humanista venezolano, son instrumentos organizadores

\footnotetext{
“¿No vale mas, que aprovechemos este momento de anarquía, para construir un edificio completo, basado en nuestra propia pronunciacion?" (Sarmiento 2007 [1843]: 30). Otro paralelismo, de temperamento: Webster "encontraba deleite en la controversia" (Mencken 1919: 251). Y Sarmiento: “ ¡Viva la polémica! Campo de la civilización en que así se baten las ideas como las preocupaciones, las doctrinas recibidas como el pensamiento y los desavíos individuales" (Sarmiento 1887: 224-225).
} 
culturales que representan, al mismo tiempo, una visión (y, como diría Unamuno, una "audición") del mundo y de la nación. Hemos considerado la ortografía, y la famosa "polémica" sobre la misma, en el contexto de la formación de la nación chilena y como una disciplina que refleja la situación social y cultural del período. Según Sebba:

[Las ortografías] no son sencillamente logros tecnológicos extraordinarios, aunque eso sí lo son. También son logros complejos sociales y culturales, mejor vistos como conjuntos de prácticas [...]. Son microcosmos de la lengua misma, donde son prominentes las cuestiones que impregnan la lengua: la historia, la identidad, la etnicidad, la cultura y la política (2007: 167).

\section{REFERENCIAS BIBLIOGRÁFICAS}

Alonso, Amado. 1972 [1951]. Introducción a los estudios gramaticales de Andrés Bello. En Andrés Bello, Gramática de la lengua castellana destinada al uso de los americanos, pp. ix-lxxxvi. Caracas: Ministerio de Educación. [= Bello, Obras completas, IV].

Annoni, Marco. 2010. Culture, Power, and Dictionaries: How to Use Lexicography to Study Cultural Objects. Semiotix XN-2. http://semioticon.com/semiotix/2010/07/ culture-powerand-dictionaries-how-to-use-the-lexicography-to-study-cultural-objects/. [Consulta: 18 de junio de 2013]

[Aster, Jane, seud.] [1863]. The Habits of Good Society. A Handbook for Ladies and Gentlemen. New York: Carleton.

Bello, AndRÉs. 1981a [1845]. Diccionario de la lengua castellana por la Academia Española (novena edición). En Obras completas, V, pp. 117-121. Caracas: La Casa de Bello.

1981b [1847]. Gramática de la lengua castellana destinada al uso de los americanos. Ed. crítica de Ramón Trujillo. Tenerife: Instituto Universitario de Lingüística Andrés Bello.

1981c [1862]. Compendio de gramática castellana escrito para el uso de las escuelas primarias. En Obras completas, Vol. V, 235-309. Caracas: La Casa de Bello.

Blanco, Mercedes Isabel. 1994. Aspectos históricos e ideológicos de las actitudes lingüísticas en el Río de la Plata y Chile (1810-1850). En María Beatriz Fontanella de Weinberg (comp.). El español en el Nuevo Mundo: Estudios sobre historia lingüistica, pp. 99-120. Washington, D. C.: OEA/OAS, Interamer, Serie Cultural 30.

Calero Vaquera, María Luisa. 2010. Ideología y discurso lingüístico: la Etnortografía como subdisciplina de la glotopolítica. Boletín de Filología 45(2): 31-48.

Cooper, Robert L. 1989. Language Planning and Social Change. Cambridge: Cambridge University Press.

Del Valle, José y Luis Gabriel-Stheeman (comps.). 2004. La batalla del idioma: La intelectualidad hispánica ante la lengua. Madrid/Frankfurt am Main: Iberoamericana/ Vervuert.

Delgado de Jesús y María, el P. Santiago. 1799 [1790]. Elementos de gramática castellana, ortografia, calografia [sic], y urbanidad, para uso de los discípulos de las Escuelas Pías. 2. impresión. Madrid: Benito Cano. 
FrieL, Brian. 1981. Translations. London: Faber and Faber.

García, Nicasio H. 1947. Plan concéntrico de ortografia. Curso elemental. 2. a ed. Montevideo: Libreros-Editores A. Monteverde y Cía.

García del Río, Juan y Andrés Bello. 1972 [1823]. Indicaciones sobre la conveniencia de simplificar i uniformar la ortografía en América. En Biblioteca americana o Miscelánea de literatura, artes y ciencias por una sociedad de americanos, Vol. I, pp. 50-62. Caracas: Edición de la Presidencia de la República en homenaje al VI Congreso de la Asociación de Academias de la Lengua.

Garvin, Paul. 1954. Literacy as problem in language and culture. Georgetown University Monograph Series on Language and Linguistics 7: 117-129.

GonzÁlez Stephan, Beatriz. 1995. Las disciplinas escriturarias de la patria: constituciones, gramáticas y manuales. Estudios: Revista de investigaciones literarias 3(5): 19-46.

Haugen, Einar. 1972. The Ecology of Language. Stanford: Stanford University Press.

JAKSIC, IvÁn. 2007. Andrés Bello: La pasión por el orden. Edición revisada y ampliada. Caracas: Bid \& Co.

Kaplan, Robert B. y Richard B. Baldauf, Jr. 1997. Language Planning from Practice to Theory. Clevedon: Multilingual Matters.

KropfF, LaURA. 2005. Activismo mapuche en Argentina: trayectoria histórica y nuevas propuestas. En Pablo Dávalos (comp.). Pueblos indígenas, estado y democracia, pp. 103-132. Buenos Aires: Consejo Latinoamericano de Ciencias Sociales (CLACSO).

Mencken, H. L. 1919. The American Language: A Preliminary Inquiry into the Development of English in the United States. New York: Alfred A. Knopf.

Narvaja de Arnoux, Elvira. 2008. Los discursos sobre la nación y el lenguaje en la formación del Estado (Chile, 1842-1862). Estudio glotopolitico. Buenos Aires: Santiago Arcos.

PAYÀs, GERTRUdIs. 2008. Tradukzión i rrebelión ortográfika. Trans 12: 15-28. [En línea: www. trans.uma.es/pdf/Trans.../t12_014-28_GPayas.pdf. Consulta: 26 de septiembre de 2013].

PÉREZ, Francisco JAVIER. 1998. Lingüística y nación: Lo nacional imaginario en las escrituras no políticas del siglo XIX venezolano. Montalbán 31: 65-84.

2000. Diccionarios, discursos etnográficos, universos léxicos. Propuestas teóricas para la comprensión cultural de los diccionarios. Caracas: Universidad Católica Andrés Bello.

2013. El diccionario y los diccionarios en la obra de Bello: Historia, teoría, crítica y elaboración lexicográficas. Ponencia presentada en el Primer Simposio de la Cátedra "Andrés Bello", Archivo Central Andrés Bello, Universidad de Chile, 29 de noviembre.

Rosenblat, Ángel. 1981. Las ideas ortográficas de Bello. En Andrés Bello, Obras completas. Vol. V, pp. ix-cxxxviii. Caracas: La Casa de Bello.

Sarmiento, Domingo F. 1887 [1842]. El comunicado del otro Quídan. En Obras de D. F. Sarmiento, Vol. I, pp. 224-227. Santiago de Chile: Imprenta Gutenberg.

2007 [1843]. Memoria (sobre ortografia americana) leída a la Facultad de Humanidades. Santiago de Chile: Imprenta de la Opinión. [www.proyectosarmiento. com.ar. Consulta: 9 de septiembre de 2013].

Sebba, Mark. 2007. Spelling and Society: The Culture and Politics of Orthography Around the World. Cambridge: Cambridge University Press.

2012. Orthography as Social Action: Scripts, Spelling, Identity and Power. En Alexandra Jaffe et al. (comps.). Orthography as Social Action: Scripts, Spelling, Identity and Power, pp. 1-19. Boston/Berlin: De Gruyter.

STRAKA, TomÁs. 2006. Para una gramática de las costumbres: Tres hipótesis sobre Andrés Bello y su tiempo. En Andrés Bello y la gramática de un Nuevo Mundo: Memorias V Jornadas de Historia y Religión, pp. 37-60. Caracas: Universidad Católica Andrés Bello. 
Study Platform on Interlocking Nationalisms. www.spinnet.eu. [Consulta: 8 de agosto de 2013].

van Dijk, Teun A. 1998. Ideología: un enfoque multidisciplinario. Barcelona: Gedisa.

Velleman, Barry L. 1995. Andrés Bello y sus libros. Caracas: La Casa de Bello. Anexos a las Obras Completas de Andrés Bello, 13.

Verdevoye, Paul. 1981. Don Andrés Bello y Domingo Faustino Sarmiento: Una polémica y una colaboración. Bello y Chile: Tercer Congreso del Bicentenario, Vol. I, pp. 103-124. Caracas: Fundación La Casa de Bello.

Weinstein, Brian. 1982. Noah Webster and the Diffusion of Linguistic Innovations for Political Purposes. International Journal of the Sociology of Language 38: 85-108.

Winchester, Simon. 1998. The Professor and the Madman: A Tale of Murder, Insanity, and the Making of the Oxford English Dictionary. New York: MJF Books. 